\title{
Identification of two laccase genes in the cultivated mushroom Agaricus bisporus
}

\author{
Caroline R. Perry,${ }^{1} \uparrow$ Melvyn Smith, ${ }^{1}$ Christine H. Britnell, ${ }^{1}$ David A. Wood ${ }^{2}$ \\ and Christopher F. Thurston ${ }^{1 *}$ \\ 'Microbial Physiology Research Group, Division of Life Sciences, King's College London, Campden Hill Road, \\ London W8 $7 A H, U K$ \\ ${ }^{2}$ Microbiology and Crop Protection Department, Horticulture Research International, Worthing Road, \\ Littlehampton, West Sussex BN17 6LP, UK
}

(Received 3 February 1993; accepted 4 March 1993)

\begin{abstract}
A cDNA library was constructed in $\lambda$ gt11 using mRNA from 11-d-old mycelium of Agaricus bisporus. Three clones containing laccase sequence were identified using an affinity-purified anti-laccase antibody. From one of these clones, a $333 \mathrm{bp}$ sequence was used to identify further cDNA clones (including one which is close to full length) and a genomic clone. The coding sequences found were of two similar but not identical versions with differences at 36 out of 520 residues of deduced amino acid sequence. The laccase genes each encode a sequence expressed as a $2.3 \mathrm{~kb}$ mRNA, specifying a 520 residue polypeptide including a 19 amino acid residue signal peptide that is absent from the $\mathbf{N}$ terminus of the mature (extracellular) protein. The coding sequence of $l c c 1$ is interrupted by 14 short introns. The $l c c 1$ and $l c c 2$ genes are not allelic as they do not segregate in uninucleate spores derived from a four-spored basidium. Comparison of the deduced amino acid sequences with that of the other fungal laccases that have been cloned, and with the very similar ascorbate oxidases from higher plants shows that whilst some sequence is absolutely conserved at and around the amino acid residues involved in copper binding, the overall sequence similarities are low.
\end{abstract}

\section{Introduction}

Laccase (polyphenol oxidase: EC 1.10.3.2) is produced very abundantly by Agaricus bisporus, constituting $2 \%$ of mycelial protein during vegetative growth (Wood, $1980 a$ ). The enzyme is found predominantly in the medium and is a glycoprotein of $65 \mathrm{kDa}$ apparent molecular mass that may exist as a dimer (Wood, 1980a; Perry et al., 1993). Although the $65 \mathrm{kDa}$ 'main polypeptide' is the form of the enzyme first revealed by in vivo labelling and also constitutes a large fraction of the purified enzyme, smaller species generated by proteolytic cleavage are always present (Perry et al.,

\footnotetext{
* Author for correspondence. Tel. (071) 333 4276; fax (071) 333 4500.

†Present address: SmithKline Beecham Pharmaceuticals, Brockham Park, Betchworth, Surrey RH3 7AJ, UK.

The nucleotide sequence data reported in this paper have been submitted to GenBank and have been assigned the accession numbers L10664 for $l c c 1$ and L10663 for $l c c 2$.
}

1993). Heterogeneity may also be a consequence of variation in the extent of glycosylation of individual laccase molecules, but this has not been verified experimentally. In vitro translation and immunoprecipitation have been used to show that laccase is formed from a nascent polypeptide of $57 \mathrm{kDa}$ apparent molecular mass (Perry et al., 1993).

During recent years the laccase gene and/or cDNA sequence has been described from four sources of which the first to be reported was the ascomycete fungus Neurospora crassa (Germann \& Lerch, 1986; Germann et al., 1988). Subsequently the sequences from another ascomycete, Aspergillus nidulans (Aramayo \& Timberlake, 1990) and two basidiomycetes, Coriolus hirsutus (Kojima et al., 1990) and Phlebia radiata (Saloheimo et al., 1991) have been published. These sequences show a common pattern, in that they all encode polypeptides of about 550 amino acid residues including an $\mathrm{N}$-terminal signal peptide. In addition, the one cysteine and 10 histidine residues involved in binding the four copper atoms found in each laccase molecule are conserved, together with a small amount of sequence around the 
four regions in which the copper ligands are clustered. It is also evident that there is some basic similarity amongst all the large blue copper proteins, that is the laccases, the plant ascorbate oxidases and the mammalian plasma protein ceruloplasmin (Germann et al., 1988; Messerschmidt \& Huber, 1990).

Studies of laccase at the molecular level in Ag. bisporus have been undertaken, not only because it is such an abundant protein in this organism, but also because its regulation of expression and activity are (at least at the physiological level) closely integrated with fruit body development (Wood \& Goodenough, 1977; Wood, $1980 b$; Smith et al., 1989).

\section{Methods}

Organism. Agaricus bisporus strain D649 was maintained on malt agar at $25^{\circ} \mathrm{C}$ and subcultured at monthly intervals. Homokaryon strains B237, B238, B239 and B240 (Loftus et al., 1988) were the complete tetrad progeny from a (rare) four-spored basidium, isolated by Elliott (1972). Cultures for mRNA or DNA isolation were grown in malt-extract liquid medium as described previously (Perry et al., 1993).

Host-vector systems. cDNA library construction employed $\lambda \mathrm{gt} 11$ in Escherichia coli Y1090 (Young \& Davis, 1983). A genomic clone was isolated from a partial genomic library of $\mathrm{Ag}$. bisporus D649 DNA (Loftus et al., 1988) in 2EMBL3 in E. coli NM392 (Frischauf et al., 1983). Subcloned sequences were manipulated in pBluescript KS (Strategene) in E. coli XL1Blue (Strategene).

$c D N A$ cloning. Poly(A)-containing RNA was isolated from the mycelium of 11-d-old Ag. bisporus cultures in malt-extract liquid medium as described previously (Perry et al., 1993). cDNA synthesis employing AMV reverse transcriptase was performed essentially as described previously (McBride \& Thurston, 1983). Double stranded cDNA was blunt-ended and ligated to EcoRI adaptors (Pharmacia) for ligation to $\lambda \mathrm{gt} 11$ arms (Amersham).

Isolation of laccase cDNA clones. Plaque lifts were screened with an affinity-purified antibody, raised against pure laccase main polypeptide (Perry et al., 1993), using phosphatase-labelled goat anti-rabbit IgG as second antibody, as described by Huynh et al. (1983).

Isolation of clones by hybridization. A fragment from one expressing clone (plcc5a, see Results and Discussion) was used as a random hexamer-primed radiolabelled probe to isolate further (non-expressing) cDNA clones and a genomic clone ( $\lambda \mathrm{lccg} 1$, see Results and Discussion) from the partial genomic DNA library made by Loftus et al. (1988) by hybridization to plaque lifts using standard methods (Sambrook et al., 1989).

Northern blot analysis. Glyoxylated RNA was analysed by electrophoresis and hybridization with plcc5a as a radiolabelled probe as described previously (Pollard et al., 1988; Thurston et al., 1988).

Sub-cloning and sequencing. Sub-cloning into pBluescript, DNA preparation and restriction analysis were performed by standard methods following Sambrook et al. (1989). Sequencing by the dideoxychain termination method (Sanger et al., 1977) was carried out on double stranded templates using Sequenase version 2 (USB) with specific chemically synthesized oligonucleotide primers where necessary. All sequence reported was determined on both strands.
$P C R$. Complete inserts of the cDNA clones in $\lambda$ gt 11 were amplified using commercially available primers (Promega) complementary to sequence either side of the cloning site in this vector with Taq polymerase (M186, Promega), for 35 cycles of $1 \mathrm{~min}$ at $95^{\circ} \mathrm{C}, 1 \mathrm{~min}$ at $50{ }^{\circ} \mathrm{C}$ and $3 \mathrm{~min}$ at $72{ }^{\circ} \mathrm{C}$, followed by one $9.9 \mathrm{~min}$ period of extension at $72{ }^{\circ} \mathrm{C}$.

Amplification of a fragment from the genomic DNA of $\mathrm{Ag}$. bisporus D649 was for 39 cycles of $1 \mathrm{~min}$ at $95^{\circ} \mathrm{C}, 1 \mathrm{~min}$ at $60^{\circ} \mathrm{C}$ and $3 \mathrm{~min}$ at $72{ }^{\circ} \mathrm{C}$, followed by one $9.9 \mathrm{~min}$ period of extension at $72{ }^{\circ} \mathrm{C}$. The primers for this reaction were complementary to a subterminal region of the $\lambda \mathrm{lccg} 1$ sequence $\left(5^{\prime}-3^{\prime}\right.$, residues $\left.2627-2645\right)$ and complementary to a subterminal region of plcc6 $\left(3^{\prime}-5^{\prime}\right.$, residues $2981-2964$, as numbered in Fig. 1 in both cases).

All PCR products were isolated by electrophoresis, blunt-ended by filling in with $\mathrm{T}_{4}$ DNA polymerase and cloned into the SmaI site of pBluescript. Sequence reported from PCR-derived clones was obtained from three independently amplified DNA fragments giving identical sequence in every case.

Sequence analysis. Sequence data were assembled using IBI Pustell MacVector software. Sequence comparison and alignment was performed using GCG programs (Devereux et al., 1984).

\section{Results and Discussion}

\section{Isolation of laccase cDNA clones}

cDNA from mRNA of 11-d-old mycelium of $\mathrm{Ag}$. bisporus was used to generate a library of about $10^{7}$ recombinants in $\lambda$ gt 11 , using $E c o$ RI adaptors. When $10^{5}$ plaques were screened with affinity-purified anti-laccase IgG, three positively reacting plaques were isolated and purified ( $\lambda \operatorname{lcc} 411, \lambda l c c 511$ and $\lambda l c c 611)$. All three recombinants were found to synthesize immunoreactive fusion proteins when converted to lysogens (data not shown). The $\lambda$ lcc511 insert was subcloned as two EcoRI fragments, plcc5a (333 bp) and plcc5b (110 bp). The plcc5a fragment contained an ORF, in-frame with the $\beta$ galactosidase coding sequence of the vector, that encoded the deduced amino acid sequence (residues 408-518 in Fig. 2), including the readily recognizable conserved laccase copper-binding motifs HisProPheHisLeuHisGlyHis(417-424)and HisCysHisIleAspTrpHis(470-476). When the sequence of plcc5b was joined to the plcc5a sequence at the end that did not match the adaptor sequence used, the ORF was continued for two amino acid residues, followed by a stop codon and $100 \mathrm{bp}$ of $3^{\prime}$ non-coding sequence. The relative orientation of these two subclones (and their contiguity) was subsequently confirmed by PCR amplification and sequencing of the complete insert of $\lambda \operatorname{lcc} 511$. The insert of $\lambda \operatorname{lcc} 411$ was 109 bp entirely included within the sequence of plcc5a (encoding amino acid residues 434 to 469 in Fig. 2, also in-frame with the vector $\beta$-galactosidase sequence). The third independently isolated cDNA clone $\lambda$ lcc611, yielded a single $E c o$ RI fragment on subcloning (plcc6, residues $2754-2841$ and $2907-3008$ in Fig. 1) that encoded the same two amino acid residues and stop 
AGGATCAACA ATG AGg CTG TCC AAC GCT TTA GTA TTG GTC GCC GCA TGC ATT TCG AGT GTA GTT GCG AAA Met Arg Leu Ser Asn Ala Leu Val Leu Val Ala Ala Cys Ile Ser Ser Val Val Ala Lys

ACC AGA ACC TTC GAC TTC GAC CTA GTC AAT ACC AGG CTC GCG CCT GAT GGG TTT GAA AGG GAgtgagtata Thr Arg Thr Phe Asp Phe Asp Leu Val Asn Thr Arg Leu Ala Pro Asp Gly phe Glu Arg As

ACA CTG ATC CAA GTC AAC AAG GGC GAC AGT GTC CGC ATC CCT CTC CAT AAC AAA CTG ACT AGC CCG ACC Thr Leu Ile Gln Val Asn Lys Gly Asp Ser Val Arg Ile pro Leu His Asn Lys Leu Thr Ser Pro Thr 60

ATG AGG CGA AGT GTA TCC ATTgtgagttacttttgagcccagattgacttgactatataacacaattagCAT TGG CAT

Met Arg Arg Ser Val Ser Ile INTRON II

GGA TTT TTC CAA GCG AGA ACT TCT GGT CAG GAC GGG CCT TCC TTC GTG AAT CAG TGC CCT CAG CCA CCC Gly Phe Phe Gln Ala Arg Thr Ser Gly Gln Asp Gly Pro Ser Phe Val Asn Gln Cys Pro Gln Pro Pro 90 100

AAC ACA ACC TTC ACC TAT GAA TTC AGC GTG GCG GAG CAG TCT GGA ACG TTCgtcctgatcccttaatacatc Asn Thr Thr Phe Thr Tyr Glu Phe Ser Val Ala Glu Gln Ser Gly Thr Phe

* $110 \quad 120$

ttcagcgtaattgacatttcttcaagttatTGG TAC CAT TCA CAC TTG TCC ACC CAA TAC TGT GAT GGT CTT CGC INTRON III Trp Tyr His Ser His Leu Ser Thr Gln Tyr Cys Asp Gly Leu Arg 130

GGT GCA TTC ATC GTT TAC GgtaattccttcctcttgcatgcgaagatatttttgccgactttcgtttagAC CCA CGT GAT Gly Ala Phe Ile Val Tyr A INTRON IV sp Pro Arg Asp

140

CCA CTC AGA CAT CTC TAT GAT GTT GAT GAC GAA AGT ACC GTC ATC ACT CTA GCT GAA TGG TAT CAC ATC pro Leu Arg His Leu Tyr Asp Val Asp Asp Glu Ser Thr Val Ile Thr Leu Ala Glu Trp Tyr His Ile $\begin{array}{lll}150 & 160 & 170\end{array}$

CTC GCA CCC GAT GCC ACT AAC GAG TTT TTC AGC TCT GGC ATT ATA CCgtaagcttct aaaggatatttattcat Leu Ala Pro Asp Ala Thr Asn Glu Phe Phe Ser Ser Gly Ile Ile Pr INTRON $\mathrm{V}$ 180

tttcataacccaattcctgcagC GTG CAA GAT TCT GGC TTG ATC AAC GGT AAA GGT AGA TTC AAT GGA GGA CCC - Val Gln Asp Ser Gly Leu Ile Asn Gly Lys Gly Arg Phe Asn Gly Gly Pro 190 200

CTC AgtaagtagatcaaaacagaaaggtgatgatgaaatttgactttacttgcttagCA CCA TTC GCC GTC GTC AAT GTT Leu T INTRON VI hr Pro Phe Ala Val Val Asn Val 210

CGC CGA GGC AAA CGT TAC AGG CTC CGT GTC ATA GCT ATT TCT TGC AGG CCG TTC TTC ACC TTC TCC GTC Arg Arg Gly Lys Arg Tyr Arg Leu Arg Val Ile Ala Ile Ser Cys Arg Pro Phe Phe Thr Phe Ser Val 220 230

GAT AAC CAT AGC TTG GTC TTC ATG GAA GCC GAT GGT GTT GAA CAT GAC CCT GTC GAA GTC CAA AAC GTC Asp Asn His Ser Leu Val phe Met Glu Ala Asp Gly Val Glu His Asp pro Val Glu Val Gln Asn Val * 240 250 260 GAC ATC TAC GCG GCC CAG CGT GTC TCG GTT ATT CTG CAT GCC AAT CAA CCC ATC GAT AAC TAC TGG ATC Asp Ile Tyr Ala Ala Gln Arg Val Ser Val Ile Leu His Ala Asn Gln pro Ile Asp Asn Tyr Trp Ile 270 280 
atgaaacgtgtttccaagitA AAC ATC AGC CTC ACT CTC GCC ATC CTG CGC TAC CAT GGT GCA CGT CAC GTC GAA 1777 eu Asn Ile Ser Leu Thr Leu Ala Ile Leu Arg Tyr His Gly Ala Arg His Val Glu * 300 310

CCG ACT ACG GTC AAC GTT CCC GGA CAC AAA CTC CTT GAT CAA GAA ATG CATgtaagaataacttgcgtaaagg

pro Thr Thr Val Asn Val Pro Gly His Lys Leu Leu Asp Gln Glu Met His 320 330

cattccgaaatacttactaacgcgcaccaatctagCCG ATC CGC CAG GAA GGA CCA GGC AAAgtgtgtatatatttaca INTRON VIII Pro Ile Arg Gln Glu Gly pro Gly Lys 340

tgaacataatcatcaactcaacgttctctagCTC GGT GAC GGT CCC CCC GAT AAG CAC ATC ACC CTC AAC ATM GCT INTRON IX Leu Gly Asp Giy Pro Pro Asp Lys His Ile Thr Leu Asn Ile Ala 350

CAAgtaagcgtctcattattcaatcaactcgacttctgacatgagcacagCCC AAC GCT CCC TTC TTC GAT ATC AAC Gln INTRON $\mathrm{X}$ Pro Asn Ala Pro Phe Phe Asp Ile Asn 360

GGA ATC TCT TAC ATC TCC CCG ACT GTC CCT GTT CTT CTC CAA ATC CTC AGC GGA GCC AAG AGG CCC GAA Gly Ile Ser Tyr Ile Ser Pro Thr Val Pro Val Leu Leu Gln Ile Leu Ser Gly Ala Lys Arg Pro Glu $370 \quad 380 \quad 390$ GAT GTT CTT CCA TCC GAA CAA ATC TTC TTT GTC CCC AAG AAT TCA TTG ATT GAA GTC AAC ATT CCA GGA Asp Val Leu Pro ser Glu Gln Ile Phe Phe Val Pro Lys Asn Ser Leu Ile Glu Val Asn Ile Pro Gly 400 410

GAA GGA GCT CAT CCA TTC CAT TgtatggtaacctcattttctcctcaattcctctacttacgtccetgtttcagTG CAC GGT Glu Gly Ala His Pro Phe His L INTRON XI eu His Gly 420

CAT AAC TTT GAT GTC GTT CTG GCT TCT AAC GAC GAC ACT TTC AAC TTC AAG AAgtgagttaattgaaaaagga His Asn Phe Asp Val Val Leu Ala Ser Asn Asp Asp Thr Phe Asn Phe Lys As 430 440

agttacctgctgaacactaatctcaatgaaagC CCG CCT CGT CGT GAC GTA TAC CCC ATC AAC GGT GGC AAC ACC INTRON XII $n$ Pro pro Arg Arg Asp Val Tyr pro Ile Asn Gly Gly Asn Thr 450

ACT TTC CGC TTC TgtaagattccacgtttcttetgaagaataactctttctgaacgacccetcagTC ACC GAT AAC CCA Thr Phe Arg Phe $\mathrm{P} \quad$ INTRON XIII he Thr Asp Asn Pro 460

GGC GCA TGG TTC TTG CAT TGgtgaggaacatttctatccatttctctcgaggcgaatactgattgaatcttgtgacatagC

Gly Ala Trp Phe Leu His Cy INTRON XIV

1850 470

CAT ATC GAT TGG CAT CTT GAA GCT GGT CTC GCT ATT GTG TTT GCT GAG GCT CCG GAG GAT AAC GTT TCA His Ile Asp Trp His Leu Glu Ala Gly Leu Ala Ile Val Phe Ala glu Ala Pro Glu Asp Asn Val Ser $480 \quad 490$ *

GGT CCC CAG TCT CAG ATC ACT CCT CAA GAT TGG TTG GAT CTC TGT CCA GAA TAC AAC GCA ATC GAG CCT Gly Pro Gln Ser Gln Ile Thr Pro Gln Asp Trp Leu Asp Leu Cys Pro Glu Tyr Asn Ala Ile Glu Pro 500 510

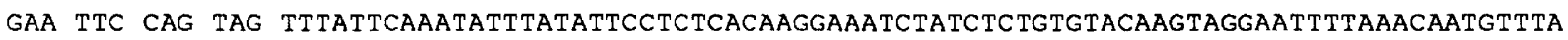
Glu Phe Gln STOP 520

CCctaaacaaatgatcgtaagtattaagtggcggcattaacgaggcaggtagtaatgat aacaaacCCAGGCACT TGATGCGCTTTGAA

Fig. 1. The nucleotide sequence of the laccase gene $l c c 1$ from $\mathrm{Ag}$. bisporus. Intron sequences are shown in lower case. Putative 'CAAT' and 'TATA' motifs in the $5^{\prime}$ non-coding region are boxed. Other motifs in the 5 ' non-coding region are: an inverted repeat between residues 153 and 199 (underlined); a 13 bp sequence homologous to residues 87-75 upstream of an ORF in the La France disease dsRNA M1 of Ag. bisporus (overline marked 'a', residues 117-129, cf. Harmsen et al., 1991); a 10 bp sequence homologous to residues -59 to -50 of the $N$. crassa laccase gene $5^{\prime}$ region (overline marked ' $b$ ', residues 177-186, cf. Germann et al., 1988); a 10 bp sequence homologous to residues -163 to -172 of the $C$. hirsutus 5 ' region (overline marked 'c', residues 297-306, cf. Kojima et al., 1990). Possible $\mathrm{N}$-glycosylation sites in the deduced amino acid sequence are shown with an asterisk. Lys $_{20}$ is the N-terminal residue of the mature extracellular protein.

codon found at the $5^{\prime}$ end of plcc5b, but was not identical with much of the $3^{\prime}$-non-coding sequence in that clone. PCR amplification of the complete insert in $\lambda l \mathrm{lcc} 611$ showed the presence of an additional insert sequence encoding the seven amino acid residues upstream of the two in the subcloned fragment (i.e. residues $512-518$ in Figs 1 and 2) again in-frame with the vector ORF.

The plasmid subclone plcc5a was used as a probe to 


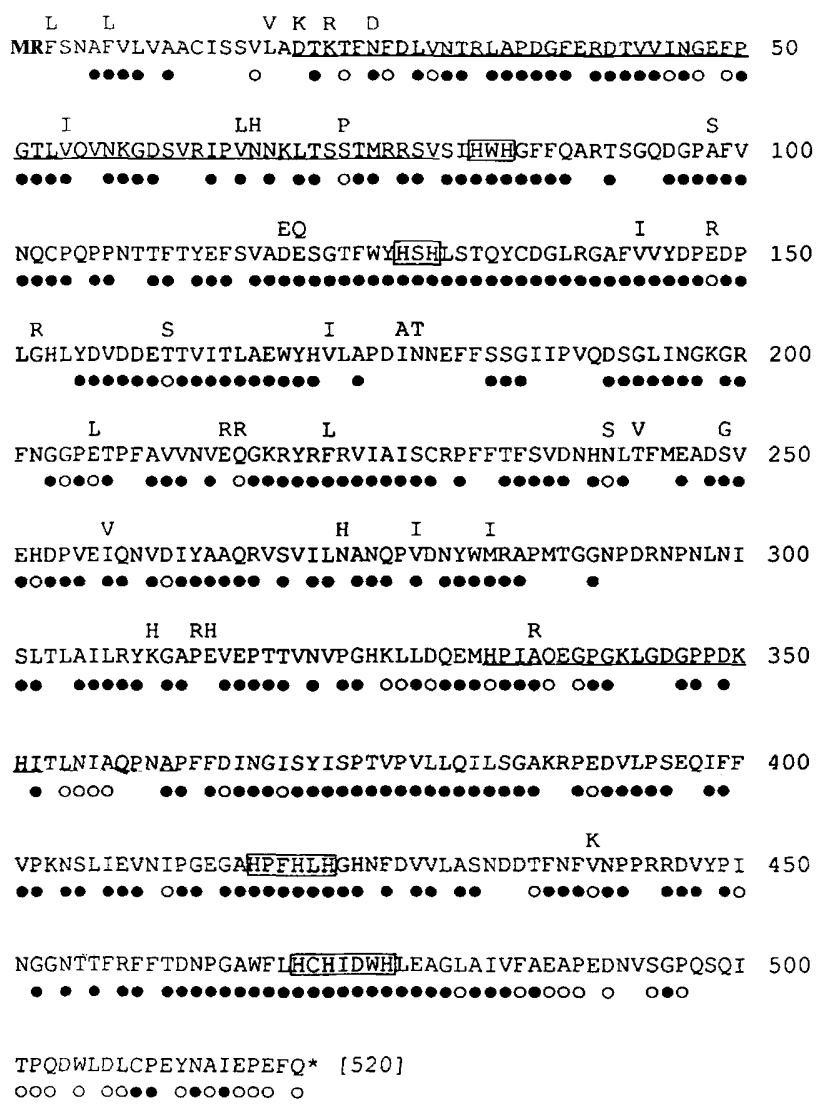

Fig. 2. Deduced amino acid sequence from the cDNA sequence of laccase gene lcc 2 of Ag. bisporus. Those residues that differ in the sequence deduced from $l c c 1$ are indicated above the line. The first two residues shown for $l c c 2$ (bold type) are extrapolated from $l c c 1$ sequence as the longest $l c c 2$ cDNA that was isolated extended only to the third base of the codon for the arginine residue after the methionine shown as initiating the sequence. The underlined sequence 20-79 was found as the chemically determined $\mathrm{N}$-terminal sequence of pure extracellular laccase main polypeptide. The underlined sequence 333-352 was found as the chemically determined $\mathrm{N}$-terminal sequence of a CNBr-fragment of pure laccase. Sequence including the 10 histidine and one cysteine residues thought to be involved in copper binding are boxed. Solid circles below the sequence indicate residues that are similar in the laccase sequences of Ag. bisporus, $C$. hirsutus and $P$. radiata; open circles indicate positions at which the Ag. bisporus sequence is similar to either that of $C$. hirsutus or that of $P$. radiata, but not both. Amino acids regarded as similar in this context were the default set in the GCG file Simplify.Txt.

isolate further clones from the cDNA library. A cDNA clone $\lambda \mathrm{lcc} 451$ (one of several apparently identical isolates) gave a $1014 \mathrm{bp} \mathrm{EcoRI} \mathrm{fragment} \mathrm{(encoding}$ residues $180-518$ in Fig. 2). PCR amplification of the complete insert of $\lambda \mathrm{lcc} 451$ showed the presence of four further EcoRI fragments. This sequence begins $5 \mathrm{bp}$ from the start of the Met codon shown as the first residue in Fig. 2 and includes $76 \mathrm{bp}$ of the $3^{\prime}$ non-coding sequence derived from plcc5b (1634 bp in all). Within this sequence two regions align with amino acid sequence determined chemically from pure laccase protein. The $\mathrm{N}$ terminus of the mature protein is identical with the sequence from $\mathrm{Asp}_{20}$ for the 60 residues determined (Fig. 2). The sequence from $\mathrm{His}_{333}$ is identical to the $\mathrm{N}$-terminal sequence of a cyanogen bromide fragment of pure laccase for 20 residues (Fig. 2).

\section{Laccase $m R N A$}

Northern blot analysis of both total RNA (Fig. 3) and the poly(A)-containing RNA fraction (not shown) from malt extract-grown $\mathrm{Ag}$. bisporus mycelium showed a single band of mRNA for laccase when probed with a laccase cDNA sequence (plcc5a). Laccase mRNA was estimated to be $2 \cdot 3 \pm 0 \cdot 15 \mathrm{~kb}$.

\section{Isolation of a genomic laccase sequence}

The plasmid subclone plcc5a was also used to probe a partial genomic library of the same strain of $\mathrm{Ag}$. bisporus (Loftus et al., 1988), from which a single reacting clone $\lambda l c c g 1$ was obtained. From subclones obtained as $K p n I$, EcoRI and Sall fragments, the sequence shown in Fig. 1 


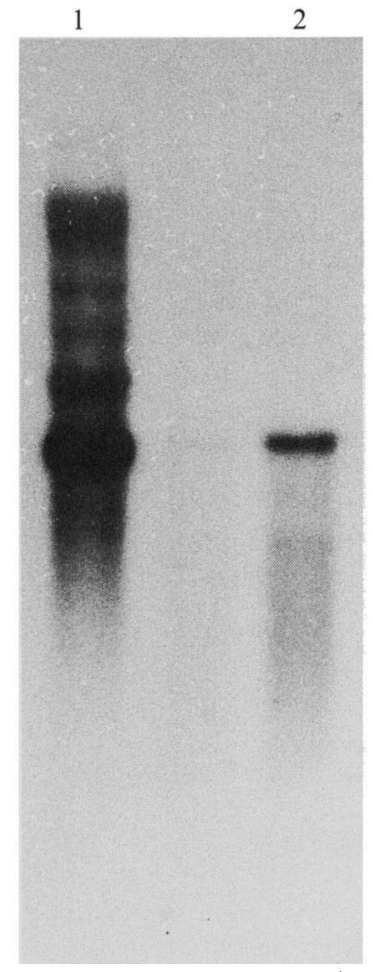

Fig. 3. Northern blot analysis of laccase mRNA. Total RNA from 7d-old $\mathrm{Ag}$. bisporus mycelium was denatured with glyoxal and separated by electrophoresis. After transfer to nitrocellulose, the laccase mRNA was located by hybridization with ${ }^{32} \mathrm{P}$-labelled plcc5a DNA (lane 2). The laccase probe also hybridized to some bands of marker HindIIIdigested $\lambda$ phage DNA (lane 1). The most heavily labelled marker band is $2 \cdot 3 \mathrm{~kb}$.

was determined, as far as residue 2701 . The remaining sequence (2702-3008) was obtained by PCR amplification from $\mathrm{Ag}$. bisporus D649 DNA (see Methods).

\section{Existence of two laccase genes}

The deduced coding sequence of the overlapping set of cDNA clones ( $\lambda \operatorname{lcc} 511, \lambda l \mathrm{lcc} 411$ and $\lambda l \mathrm{lcc} 451)$ differed from the cDNA clone $\lambda 611$ and the sequence found as exons in the genomic sequence at 36 out of 520 residues. The genomic sequence is named $l c c 1$ and is shown in Fig. 1. The sequence that we have described only in cDNA is named as $l c c 2$ and its deduced coding sequence is shown in Fig. 2, together with indication of those residues that differ in the coding sequence of $l c c 1$ (at the nucleic acid level, $l c c 1$ and $l c c 2$ differ at 135 of 1563 bp or $8.6 \%$ of sequence).

Ag. bisporus strain D649 is a heterokaryon carrying at least two nuclear types, such that the two laccase sequences we have detected could be alleles at the same genetic locus. That this is not so has been established by analysis of a tetrad of homokaryotic strains, isolated by Elliott (1972) from a single four-spored basidium. These four haploid strains all show the same pattern of bands in Southern blot analysis with a laccase probe that binds both $l c c 1$ and $l c c 2$ sequence (Fig. 4). If the two genes were allelic, two distinct patterns would have segregated $2: 2$ amongst the four strains, as shown with the control probe $\lambda$ Ab22 (Loftus et al., 1988). Further, in a cosmid library of genomic DNA from $\mathrm{Ag}$. bisporus strain C-54carb-8 (a fungicide-resistant mutant; Loftus et al., 1988), restriction mapping using the same laccase probe showed the presence of more than one gene sequence in several independently isolated clones (Sodhi, 1992).

\section{Structure of the lccl gene}

The coding sequence of $l c c 1$ is interrupted by 14 short introns $(48-60 \mathrm{bp})$ that start with the consensus sequence GTNN(G/T) and end with (T/A/C)A(G/T). Eleven of the 14 have an internal ACT sequence $10-20 \mathrm{bp}$ from $3^{\prime}$ splicing site. These structures are generally in accord with the intron structure of the cell gene and glyceraldehyde-3-phosphate dehydrogenase genes of $\mathrm{Ag}$. bisporus (Raguz et al., 1992; Harmsen et al., 1992). The fifteenth intron ( $66 \mathrm{bp}$ ) is in the $3^{\prime}$ non-coding sequence and does not obviously exhibit any of the above consensus features.

Upstream of the coding sequence, seven sequence motifs of possible significance have been noticed. First, two possible TATA box and one CAAT element are present (boxed in Fig. 1). Secondly, there are three sequences showing homology to putative promoter region sequence found elsewhere. Two are short regions of homology to promoter region sequence of other fungal laccase genes (see Fig. 1) and the third is a region of homology to sequence $5^{\prime}$ to an ORF in the M1 dsRNA associated with La France disease of $\mathrm{Ag}$. bisporus (Harmsen et al., 1991). Thirdly, the sequence 153-200 contains an inverted repeat of 16 out of 19 residues. Within the $5^{\prime}$ non-coding region there are nine further matches to transcription factor recognition sequences of vertebrates (Faisst \& Meyer, 1992) or higher plants (Katagiri \& Chua, 1992) if comparison of 6 or $7 \mathrm{bp}$ sequences is made, but no significance can presently be attributed to any such homology. We have not identified any homology with the promoter region of the cell gene or the glyceraldehyde-3-phosphate dehydrogenase genes of Ag. bisporus (Raguz et al., 1992; Harmsen et al., 1992), other than that these genes also have putative TATA and CAAT box sequences.

\section{The deduced amino acid sequence of $l c c 1$ and $l c c 2$}

The coding sequence of $l c c 1$ is 520 amino acid residues of which the first 19 are signal peptide cleaved during export. The $\mathrm{N}$-terminal sequence of the purified extra- 
(a)

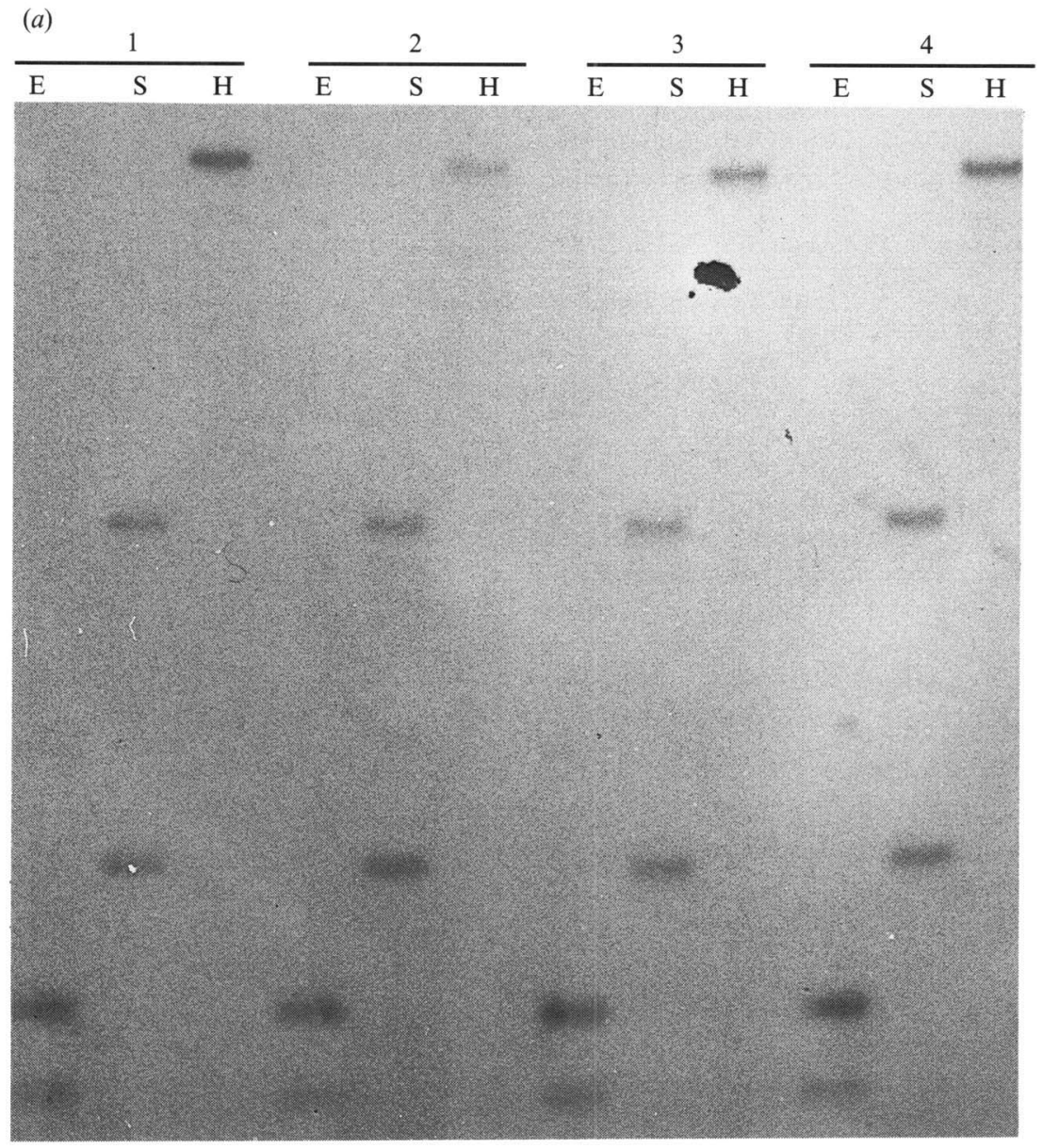

(b)
Fig. 4. Southern blot analysis of DNA from a set of homokaryotic strains of $\mathrm{Ag}$. bisporus, derived from a single four-spored basidium, probed with a laccase genomic DNA fragment $(a)$ and an anonymous $\mathrm{Ag}$. bisporus RFLP probe $\lambda \mathrm{Ab} 22(b)$. Lanes 1-4 contain DNA from strains B237, B238, B239 and B240, respectively. In (a) DNA was restricted with EcoRI (E), SalI (S) and HindIII (H). In (b), digests were with EcoRI only. The laccase probe gives identical patterns for all four strains, whereas the control probe $\lambda \mathrm{Ab} 22$ gives two distinct patterns ( 1 and 3 are different from 2 and 4 in $b$ ). 
Table 1. Percentage similarity of laccase and ascorbate oxidase sequences

Sequence similarity was derived using the GCG program PILEUP, with which gaps are allowed in aligned sequences. The nucleic acid alignments were for exon sequence only. For comparison of deduced amino acid sequence, the amino acids regarded as similar were the default set in the GCG file Simplify.Txt. Asc, Ascorbate.

\begin{tabular}{|c|c|c|c|c|c|c|c|}
\hline & N. crassa & $\begin{array}{c}\text { Asp. } \\
\text { nidulans }\end{array}$ & C. hirsutus & $P$. radiata & $\begin{array}{c}\text { Ag. } \\
\text { bisporus }\end{array}$ & $\begin{array}{l}\text { Asc. oxidase } \\
\text { (squash) }\end{array}$ & $\begin{array}{l}\text { Asc. oxidase } \\
\text { (cucumber) }\end{array}$ \\
\hline & \multicolumn{7}{|c|}{ Deduced protein sequence alignment } \\
\hline N. crassa & & $46 \cdot 9$ & $55 \cdot 1$ & $53 \cdot 2$ & $52 \cdot 8$ & $49 \cdot 9$ & $52 \cdot 0$ \\
\hline Asp. nidulans & $38 \cdot 7$ & & $48 \cdot 8$ & $48 \cdot 1$ & $52 \cdot 4$ & $51 \cdot 7$ & $45 \cdot 1$ \\
\hline C. hirsutus & $41 \cdot 6$ & $38 \cdot 3$ & & $77 \cdot 2$ & $66 \cdot 4$ & $51 \cdot 6$ & $53 \cdot 7$ \\
\hline$P$. radiata & $38 \cdot 2$ & $39 \cdot 2$ & $57 \cdot 6$ & & $64 \cdot 7$ & $53 \cdot 4$ & $51 \cdot 5$ \\
\hline Ag. bisporus & $38 \cdot 7$ & $38 \cdot 7$ & $45 \cdot 3$ & $44 \cdot 8$ & & 53.9 & $51 \cdot 5$ \\
\hline Asc. oxidase (squash) & $39 \cdot 1$ & $37 \cdot 0$ & $40 \cdot 6$ & $42 \cdot 6$ & $38 \cdot 6$ & & $88 \cdot 5$ \\
\hline \multirow[t]{2}{*}{ Asc. oxidase (cucumber) } & $38 \cdot 5$ & $38 \cdot 4$ & $36 \cdot 7$ & $41 \cdot 8$ & $38 \cdot 7$ & $73 \cdot 1$ & \\
\hline & \multicolumn{7}{|c|}{ Nucleic acid sequence alignment } \\
\hline
\end{tabular}

cellular protein matches the sequence of $l c c 2$ and not $l c c 1$ at all seven positions that differ within the region for which amino acid sequence was chemically determined (Fig. 2). Either the mature protein translated from $l c c 1$ is $\mathrm{N}$-terminally blocked or the extent of expression of $l c c 1$ is significantly less than that of $l c c 2$, although some expression of $l c c 1$ is expected as the sequence was detected as cDNA.

The encoded polypeptides have calculated $M_{\mathrm{r}}$ values of $58118.8 \mathrm{Da}(l c c 1)$ and $57827.4(l c c 2)$ and isoelectric points of 5.79 and 4.72 , respectively. The size of the nascent polypeptide obtained by in vitro translation and immunoprecipitation was estimated to be $57 \mathrm{kDa}$ (Perry et al., 1993) which is in accord with the above calculated mass values. The calculated isoelectric $\mathrm{pH}$ of the products of $l c c 1$ and $l c c 2$ differ by a readily detectable amount, but as extracellular laccase focuses as a diffuse band at $\mathrm{pH}$ 3.4 4.0 (Wood, 1980a), the glycosylated enzyme is significantly more negatively charged than calculated for either of the non-glycosylated polypeptide(s). Both $l c c 1$ and $l c c 2$ products have five potential $N$-glycosylation sites (Fig. 1). Interestingly, the substitutions $\operatorname{Ser}_{239}$ and $\mathrm{Val}_{241}$ in the $l c c 1$ product compared with the $l c c 2$ product, corrupt the potential glycosylation site at $\mathrm{Asn}_{239}$ and create an alternative site at $\mathrm{Asn}_{241}$.

Codon usage in both genes is similar showing a strong preference for third position C ( 40.5 and $42.2 \%)$, marked preference against third position $\mathrm{G}(14.8$ and $14.6 \%)$ and against third position A (16.9 and $16.3 \%$; data for $l c c 1$ and $l c c 2$, respectively). This codon usage is similar to that found for the transcribed Ag. bisporus glyceraldehyde-3phosphate dehydrogenase gene, in preference for third position $\mathrm{C}$ and in preference against $\mathrm{G}$ and $\mathrm{A}$ (Harmsen et al., 1992). The cell gene of Ag. bisporus has comparable preference against $\mathrm{G}$ and $\mathrm{A}$, but not the same preference toward $\mathrm{C}$ as $\mathrm{T}$ is the preferred third base in this gene (Raguz et al., 1992).

\section{Homology of lccl and lcc2 with other laccase and ascorbate oxidase sequences}

Laccase sequence from $\mathrm{Ag}$. bisporus was compared with laccase from Neurospora crassa (Germann et al., 1988), Aspergillus nidulans (Arayamo \& Timberlake, 1990), Coriolus hirsutus (Kojima et al., 1990) and Phlebia radiata (Saloheimo et al., 1991), together with sequence of ascorbate oxidase from cucumber (Ohkawa et al., 1989) and squash (Messerschmidt et al., 1989). Pairwise alignments of all combinations of the above sequences both as amino acid sequence similarity and as nucleic acid sequence for exons of the coding sequences are shown in Table 1. It is evident that the three basidiomycete laccases are more similar than any other combination of sequences, except for the close similarity of the two ascorbate oxidases. It is of note that the two ascomycete laccases are not closely similar to each other and are not any more similar to the other fungal laccases than are the plant ascorbate oxidases. Of the five potential $\mathrm{N}$-glycosylation sites in $\mathrm{Ag}$. bisporus laccase, only one $\left(\mathrm{Asn}_{454}\right)$ is conserved in the sequence of laccase from $P$. radiata and $C$. hirsutus. All the fungal laccases except that of Asp. nidulans conserve the two pairs of cysteines involved in intra-subunit disulphide bond formation in the ascorbate oxidases $\left(\mathrm{Cys}_{103}-\mathrm{Cys}_{509}\right.$ and $\mathrm{Cys}_{135}-\mathrm{Cys}_{229}$ in the $A$. bisporus laccase, see Fig. 2) and multiple alignment analysis (not shown) identifies the Asp. nidulans laccase as the least similar of the sequences.

One feature of the laccase sequences that completely defies explanation with current knowledge is the variability in presence of introns from $N$. crassa with one, Asp. nidulans with five, $P$. radiata with nine, $C$. hirsustus with 11 and $A g$. bisporus with 15. Exceptionally large numbers of small introns may be a characteristic feature of $\mathrm{Ag}$. bisporus, as its glyceraldehyde-3-phosphate dehydrogenase genes contain nine introns whereas the 
equivalent genes in Schizophyllum commune and Phanerochaete chrysosporium contain only five and six introns, respectively (Harmsen et al., 1992).

As the primary structure of the $N$. crassa and $P$. radiata laccases are both consistent with a $\beta$-barrel structure as found crystallographically for ascorbate oxidase (Messerschmidt \& Huber, 1990; Saloheimo et al., 1991), it is probable that all these enzymes have a similar three-dimensional architecture, notwithstanding their relatively low similarity of primary structure. Whether the basidiomycete enzymes are more similar because of more recent evolutionary divergence or because their functions are more similar remains unresolved.

Although allelic sequence differences have been demonstrated for laccase genes both in $N$. crassa (Germann et al., 1988) and C. hirsutus (Kojima et al., 1990), this is the first report of a gene family for laccases, although multiplicity of laccase enzymes at the biochemical level has already been observed widely in the fungi (e.g., Esser \& Minuth, 1970; Marbach et al., 1984; Rehman \& Thurston, 1992). Within the genome of Ag. bisporus, two tandemly linked glyceraldehyde-3-phosphate dehydrogenase genes have been identified (of which one is apparently a non-transcribed pseudogene; Harmsen et al., 1992); these and our report of the cell gene (Raguz et al., 1992) are the only other published Ag. bisporus nuclear gene sequences at present.

We most gratefully acknowledge advice from Dr Konrad Lerch and Dr Roberta Farrell, during the early stages of this work. We thank Dr Lerch and Dr Markku Saloheimo for sending us sequence data in advance of publication. We thank Dr Tim Elliott for the gift of homokaryon strains and genomic library, and Dr Phil Cunningham for his excellent guidance with computing. Financial support from the Agricultural and Food Research Council of the UK is also gratefully acknowledged.

\section{References}

Aramayo, R. \& Timberlake, W. E. (1990). Sequence and molecular structure of the Aspergillus nidulans yA (laccase 1) gene. Nucleic Acids Research 18, 3415.

DevereuX, J., Haeberli, P. \& Smithies, O. (1984). A comprehensive set of sequence analysis programs for the VAX. Nucleic Acids Research 12, 387-395.

Elliotr, T. J. (1972). Sex and the single spore. Mushroom Science 8, $11-18$.

ESSER, K. \& MinUTH, W. (1970). The phenoloxidases of the ascomycete Podospora anserina VI. Genetic regulation of the formation of laccase. Genetics 64, 441-458.

FAISST, S. \& MEYER, S. (1992). Compilation of vertebrate-encoded transcription factors. Nucleic Acids Research 20, 3-26.

Frischauf, A. M., Lehrach, H., Poutska, A. \& Murray, N. (1983). Lambda replacement vectors carrying polylinker sequences. Journal of Molecular Biology 170, 827-842.

GermanN, U. A. \& LeRCH, K. (1986). Isolation and partial nucleotide sequence of the laccase genes from Neurospora crassa: amino acid sequence homology of the protein to human ceruloplasmin. Proceedings of the National Academy of Sciences of the United States of America 83, 8854-8858.

Germann, U. A., Muller, G., Hunziker, P. E. \& Lerch, K. (1988) Characterisation of two allelic forms of Neurospora crassa laccase. Amino- and carboxyl-terminal processing of a precursor. Journal of Biological Chemistry 263, 885-896.

Harmsen, M. C., Tolner, B., Kram, A., Seung, J. G., de HaAn, A. \& Wessels, J. G. H. (1991). Sequences of three dsRNAs associated with La France disease of the cultivated mushroom (Agaricus bisporus). Current Genetics 20, 137-144.

Harmsen, M. C., Schuren, F. H. J., Moukha, S. M., van Zuilen, C. M., Punt, P. J. \& Wessels, J. G. H. (1992). Sequence analysis of the glyceraldehyde-3-phosphate dehydrogenase genes from Schizophyllum commune, Phanerochaete chrysosporium and Agaricus bisporus. Current Genetics 22, 447-454.

Huynh, T. V., Young, R. A. \& Davis, R. W. (1983). Construction and screening of cDNA libraries in $\lambda \mathrm{gt} 10$ and $\lambda \mathrm{gt} 11$. In DNA Cloning. $A$ Practical Approach, pp 49-78. Edited by D. M. Glover. Oxford: IRL Press.

KatagiRI, F. \& ChUA, N.-H. (1992). Plant transcription factors: present knowledge and future challenges. Trends in Genetics $\mathbf{8}$, 22-27.

Kojima, Y., Tsukuda, Y., Kawai, Y., Tsukamoto, A., Sugiura, J., SAKaIMo, M. \& KITA, Y. (1990). Cloning, sequence analysis, and expression of lignolytic phenoloxidase genes of the white-rot basidiomycete Coriolus hirsutus. Journal of Biological Chemistry 265, 15224-15230.

Loftus, M. G., Moore, D. \& Elliott, T. J. (1988). DNA polymorphism in commercial and wild strains of the cultivated mushroom, Agaricus bisporus. Theoretical and Applied Genetics 76, $712-718$.

MCBride, N. K. \& Thurston, C. F. (1983). The complexity of Poly(A) ${ }^{+}$RNA in the alga Chlorella fusca. Journal of Cell Science 63, 223-231.

Marbach, I., Harel, E. \& MaYeR, A. L. (1984). Molecular properties of extracellular Botrytis cinerea laccase. Phytochemistry 23, 2713-2717.

Messerschmidt, A. \& HubER, R. (1990). The blue oxidases, ascorbate oxidase, laccase and ceruloplasmin. Modelling and structural relationships. European Journal of Biochemistry 187, 341-352.

Messerschmidt, A., Rossi, A., Ladenstein, R., Huber, R., Bolognesi, M., Gati, G., Marchesini, A., Petruzelli, R. \& Finazzi-Agro, A. (1989). X-ray crystal structure of the blue oxidase ascorbate oxidase from Zucchini. Analysis of the polypeptide fold and a model of the copper sites and ligands. Journal of Molecular Biology 206, $513-529$

Ohkawa, J., Okada, N., Shinmyo, A. \& Takano, M. (1989). Primary structure of cucumber (Cucumis sativus) ascorbate oxidase deduced from cDNA sequence: homology with blue copper proteins and tissue-specific expression. Proceedings of the National Academy of Sciences of the United States of America 86, 1239-1243.

Perry, C. R., Matcham, S. E., Wood, D. A. \& Thurston, C. F. (1993). The structure of laccase protein and its synthesis by the commercial mushroom Agaricus bisporus. Journal of General Microbiology 139, 171-178.

Pollard, J. W., Perry, C. R. \& Thurston, C. F. (1988). Northern blotting. In Methods of Molecular Biology, vol. 3, pp. 13-26. Edited by J. Walker. Clifton: Humana Press Inc.

Raguz, S., Yague, E., Wood, D. A. \& Thurston, C. F. (1992). Isolation and characterisation of a cellulose-growth-specific gene from Agaricus bisporus. Gene 119, 183-190.

Rehman, A. U. \& Thurston, C. F. (1992). Purification of laccase I from Armillaria mellea. Journal of General Microbiology 138, $1251-1257$.

Saloheimo, M., Niku-Paavola, M.-L. \& Knowles, J. K. C. (1991) Isolation and structural analysis of the laccase gene from the lignindegrading fungus Phlebia radiata. Journal of General Microbiology 137, 1537-1544.

Sambrook, J., Fritsch, E. F. \& Maniatis, T. (1989). Molecular Cloning. A Laboratory Manual, 2nd edn. Cold Spring Harbor, NY: Cold Spring Harbor Laboratory. 
SANGer, F., Nicklen, S. \& Coulson, A. R. (1977). DNA Sequencing with chain terminating inhibitors. Proceedings of the National Academy of Sciences of the United States of America 74, 5463-5467. Smith, J. F., Claydon, N., love, M. E., Allan, M. \& Wood, D. A. (1989). Effect of substrate depth on extracellular endocellulase and laccase production of Agaricus bisporus. Mycological Research 93, 292-296.

5ODHI, H.S. (1992). Enzyme mutants of Coprinus bilanatus and recombinant DNA technology for strain improvement in Agaricus bisporus. $\mathrm{PhD}$. thesis, University of London.

Thurston, C. F., Perry, C. R. \& Pollard, J. W. (1988). Electrophoresis of RNA denatured with glyoxal or formaldehyde. In Methods of Molecular Biology, vol. 3, pp. 3-12. Edited by J. Walker. Clifton: Humana Press Inc.
WooD, D. A. $(1980 a)$. Production, purification and properties of extracellular laccase of Agaricus bisporus. Journal of General Microbiology 117, 327-338.

WooD, D. A. $(1980 \mathrm{~b})$. Inactivation of extracellular laccase during fruiting of Agaricus bisporus. Journal of General Microbiology 117, 339-345.

Wood, D. A. \& Goodenough, P. W. (1977). Fruiting of Agaricus bisporus. Changes in extracellular enzyme activities during growth and fruiting. Archives of Microbiology 114, 161-165.

YounG, R. A. \& Davis, R. W. (1983). Yeast polymerase II genes: isolation with antibody probes. Science 222, 778-782. 